\title{
Overexpression of RhoGDI2 correlates with the progression and prognosis of pancreatic carcinoma
}

\author{
BIN YI $^{1 *}$, YI ZHANG ${ }^{1 *}$, DONGMING ZHU ${ }^{1 *}$, LIFENG ZHANG ${ }^{1 *}$, SHIDUO SONG ${ }^{1}$, \\ SONGBING HE ${ }^{1,2}$, BING ZHANG ${ }^{3}$, DECHUN LI ${ }^{1}$ and JIAN ZHOU ${ }^{1}$ \\ ${ }^{1}$ Department of General Surgery, The First Affiliated Hospital of Soochow University, Suzhou, Jiangsu 215006; \\ ${ }^{2}$ Shanghai Institute of Immunology, Shanghai Jiao Tong University School of Medicine, Shanghai 200025; \\ ${ }^{3}$ Department of Nuclear Medicine, The First Affiliated Hospital of Soochow University, \\ Suzhou, Jiangsu 215006, P.R. China
}

Received October 2, 2014; Accepted December 11, 2014

DOI: 10.3892/or.2015.3707

\begin{abstract}
Rho GDP dissociation inhibitor 2 (RhoGDI2) has been found to be a regulator of tumor metastasis. However, the expression of RhoGDI2 and its clinicopathological significance as well as the pathway of RhoGDI2 in tumor metastasis have yet to be investigated. To investigate the role of RhoGDI2 in the progression and prognosis of pancreatic carcinoma (PC), the expression of RhoGDI2 in human PC tissues was examined and compared with the clinicopathological characteristics and prognosis. Moreover, the relationship between RhoGDI2 and E-cadherin was examined. The results indicated that RhoGDI2 was overexpressed in PC tissues and associated with clinicopathological characteristics, including clinical stage and lymph node metastasis. Patients with a RhoGDI2-negative expression had a significantly longer survival time than those with a RhoGDI2-positive expression. Additionally, the expression of RhoGDI2 was negatively correlated with the expression of E-cadherin in PC tissues. Taken together, the findings suggest that RhoGDI2 is important in the progression and prognosis of $\mathrm{PC}$, and may be used as a potential prognostic biomarker and a therapeutic target for PC.
\end{abstract}

\section{Introduction}

Human pancreatic carcinoma (PC) is a highly aggressive malignant cancer with a poor prognosis. Numerous treatment protocols have been applied to PC, however, the 5-year survival rate remains $<5 \%$, partly due to $\mathrm{PC}$ cells being resistant to chemotherapy and radiation $(1,2)$. Vascular invasion

Correspondence to: Dr Jian Zhou, Department of General Surgery, The First Affiliated Hospital of Soochow University, 188 Shizi Street, Suzhou, Jiangsu 215006, P.R. China

E-mail: zhoujian0612@gmail.com

${ }^{*}$ Contributed equally

Key words: RhoGDI2, E-cadherin, pancreatic carcinoma, prognosis and distant metastasis are the critical features in the aggressive phenotype of PC, and contribute to the principal causes of PC deaths. Thus, biomarkers associated with the invasion and metastasis and survival of PC are required to predict patient prognosis and to aid in the design of effective target therapy.

Rho GTPases, including Rac1, Cdc42 and RhoC, are involved in the regulation of cell migration, cell motility, cell cycle progression and cytoskeleton organization $(3,4)$. Aberrant signaling of these proteins is commonly observed in many types of human cancer and is associated with aggressive phenotype. The biological activities of GTPases are regulated by guanine nucleotide exchange factors (GEFs), GTPase-activating proteins (GAPs) and Rho GDP dissociation inhibitors (RhoGDIs) (5). Rho GDP dissociation inhibitor 2 (RhoGDI2), which belongs to a family of RhoGDIs, is verified to be differentially expressed in human cancers $(6,7)$. Accumulating evidence has shown that RhoGDI 2 acts as a positive or negative regulator of cancer progression depending on the tumor type (8). In a previous study, we showed that RhoGDI 2 promoted PC cell invasion and metastasis in vitro (9). However, the expression of RhoGDI2 and its correlation with poor prognosis in PC patients as well as the pathway of RhoGDI 2 in tumor metastasis remain to be examined.

Epithelial to mesenchymal transition (EMT) is a critical morphologic conversion during tumor progression and results in the promotion of cell motility, invasion and metastasis (10). Increasing evidence suggests that EMT occurs in several types of cancer, including colorectal cancer (11), gastric cancer (12), and breast cancer (13). Loss of expression of the epithelial cell adhesion molecule E-cadherin is a prerequisite of EMT.

In this study, we examined the expression of RhoGDI2 in human PC tissues, and compared it with the clinicopathological characteristics and prognosis. Moreover, we investigated the association between RhoGDI2 and E-cadherin, a critical factor of EMT, and determined the possible pathway that RhoGDI2 may be involved in the aggressive phenotype of PC.

\section{Materials and methods}

Clinical samples. Tissue samples were collected from $77 \mathrm{PC}$ patients during surgical resections performed at the 
First Affiliated Hospital of Soochow University between January, 2008 and December, 2010. Tumorous tissues and adjacent non-tumorous tissues (NT) were frozen immediately after surgical removal in liquid nitrogen and stored at $-80^{\circ} \mathrm{C}$. The patients had not received any preoperative chemo-, radioor immunotherapy. Grades of differentiation and clinical stage were classified according to the World Health Organization. All the samples were obtained following patient consent and approval by the Ethics Committee of Soochow University.

Immunohistochemistry (IHC). The samples were fixed with formalin, embedded in paraffin and sliced. Serial sections $(4 \mu \mathrm{m})$ subjected to immunohistological staining were fixed with freshly prepared $3 \% \mathrm{H}_{2} \mathrm{O}_{2}$ with $0.1 \%$ sodium azide to quench endogenous peroxidase and then treated with antigen retrieval solution for $15 \mathrm{~min}$. After placing in blocking reagent for $15 \mathrm{~min}$, the sections were incubated in primary antiRhoGDI2 or anti-E-cadherin monoclonal antibody overnight at $4^{\circ} \mathrm{C}$, followed by incubation with the secondary antibody and Extravidin-conjugated horseradish peroxidase. The staining intensity was scored as: 0 , negative; 1 , weak; 2 , medium and 3 , strong. The extent of staining was scored as: $0,0 \% ; 1,1-25 \%$; $2,26-50 \% ; 3,51-75 \%$ and $4>76 \%$. The final score was obtained by the sum of the intensity score and the quantity score. A score of $\geq 3$ was considered as positive expression, while a score of $\geq 6$ was considered as strong-positive expression.

$R T-P C R$. Total RNA from samples was extracted by TRIzol (Invitrogen, Carlsbad, CA, USA). Total RNA $(10 \mu \mathrm{g})$ was used to synthesize single-stranded cDNA for a PCR template by reacting with random primers and M-MLV reverse transcriptase (Promega, Madison, WI, USA). The relative expression of RhoGDI2 mRNA transcripts to that of the control ( $\beta$-actin) was determined by RT-PCR. The primers used were: RhoGDI2 (606 bp) forward, 5'-ATGACTGAAAAAGCC CCA-3' and reverse, 5'-TCATTCTGTCCACTCCTT-3'; $\beta$-actin (308 bp) forward, 5'-AGCGGGAAATCGTGCGTG-3' and reverse, 5'-CAGGGTACATGGTGGTGCTGCC-3'. The PCR amplification was 40 cycles $\left(95^{\circ} \mathrm{C}\right.$ for $15 \mathrm{sec}, 62^{\circ} \mathrm{C}$ for $45 \mathrm{sec}$ and $72^{\circ} \mathrm{C}$ for $30 \mathrm{sec}$ ). The amplified segments were analyzed by $2.5 \%$ agarose gels.

Western blotting. Tissues were lysed in lysis buffer on ice. Total proteins were separated by 5-12\% SDS-PAGE and transferred onto PVDF membrane. The membrane as placed in a TBST solution with 5\% non-fat milk powder for $1 \mathrm{~h}$ at room temperature and incubated at $4^{\circ} \mathrm{C}$ overnight with primary antibodies: anti-RhoGDI2 antibody (1:200) ), anti-E-cadherin antibody (1:400; both from Abcam, Cambridge, UK) and anti- $\beta$-actin antibody (1:200), followed by incubation at room temperature for $1 \mathrm{~h}$ with a goat anti-mouse $\operatorname{IgG}(1: 2,000$, both from Santa Cruz Biotechnology, Inc., Santa Cruz, CA, USA), conjugated with horseradish peroxidase. Reactive bands were detected using ECL western blotting detection reagent.

Statistical analysis. SPSS version 17.0 was used for statistical analysis. Data were presented as mean $\pm \mathrm{SD}$. The t-test and Chi-square test were performed for inter-group comparison. The correlation between RhoGDI2 and E-cadherin expression was determined by the Pearson correlation analysis. Survival
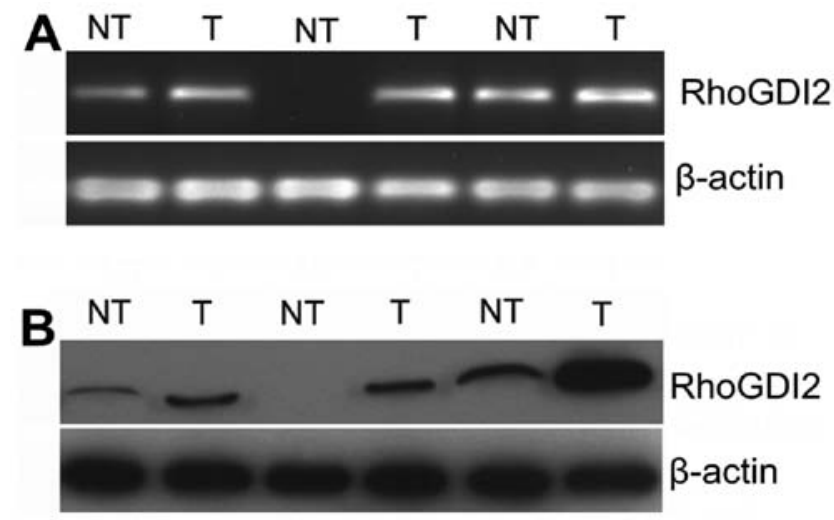

Figure 1. Expression of RhoGDI2 mRNA and protein in pancreatic carcinoma (PC) tissues and non-tumorous tissues (NT). (A) Expression of RhoGDI2 mRNA was examined in PC and NT by RT-PCR. (B) Expression of RhoGDI2 protein was examined in PC and NT by western blotting.

was assessed according to the Kaplan-Meier method and compared using the log-rank test. Multivariate analysis of prognostic markers was performed with the Cox proportional hazards regression model. $\mathrm{P}<0.05$ was considered to indicate a statistically significant difference.

\section{Results}

Expression of RhoGDI2 in PC tissues. To investigate the expression pattern of RhoGDI2 in clinical fresh PC tissues, RT-PCR and western blotting were used in 20 paired PC tissues and adjacent non-tumorous tissues. The expression of RhoGDI2 in PC tissues was higher than that in non-tumorous tissues at the mRNA level $(\mathrm{P}<0.05)$ (Fig. 1). Similarly, western blotting results revealed that the expression of RhoGDI2 protein was upregulated in PC tissues compared with that in non-tumorous tissues (Fig. 2, $\mathrm{P}<0.05$ ). These results indicated that RhoGDI2 was overexpressed in PC tissues.

Correlation between RhoGDI2 expression and clinicopathological parameters. To elucidate the role of RhoGDI2 in the progression of PC, we detected the expression of RhoGDI2 protein in PC tissues by IHC staining. The subcellular location of RhoGDI2 protein was observed mainly in the cytoplasm of cancer cells in PC tissues (Fig. 2). Among $77 \mathrm{PC}$ tissues, 49 cases (63.6\%) exhibited a positive expression of RhoGDI2, including 31 strong-positive cases (40.3\%) in tumor tissues. Among the non-tumorous tissues, there were 66 RhoGDI2-negative expression (85.7\%) and 11 weak-positive expression (14.3\%) cases, showing a significant difference $\left(\chi^{2}=39.428, P=0.001\right)$. The association between RhoGDI2 expression and clinicopathological parameters showed that RhoGDI2 expression was significantly correlated with clinical stage $\left(\chi^{2}=19.983\right.$, $\mathrm{P}=0.008)$ and lymph-node metastasis $\left(\chi^{2}=16.418, \mathrm{P}=0.013\right)$, but did not show a statistically significant association with gender, age, tumor location, tumor size and differentiation $(\mathrm{P}>0.05$, Table I) . These results indicated that the overexpression of RhoGDI2 may be correlated with the progression of PC.

Correlation between RhoGDI2 expression and PC patient prognosis. Among 77 PC patients, the follow-up success rate 

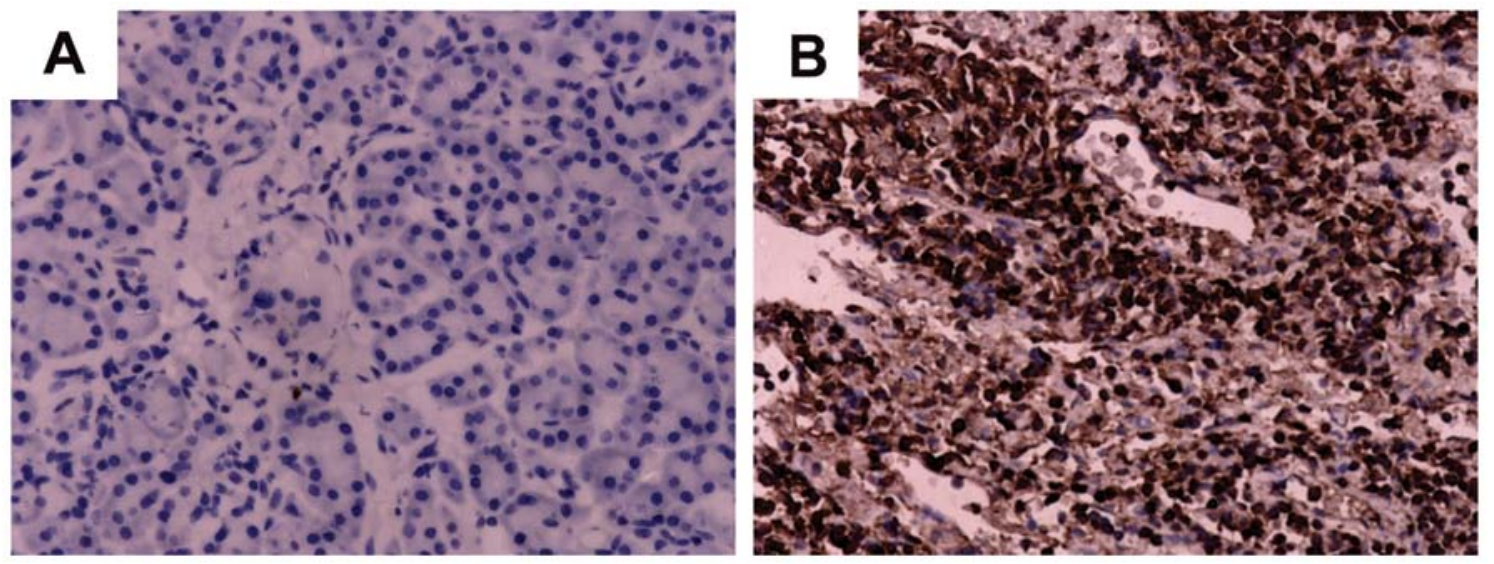

Figure 2. Immunohistochemical staining of RhoGDI2 protein. (A) Low expression in pancreatic non-tumorous tissues. (B) High expression in pancreatic carcinoma tissues. Original magnification, x200.

Table I. Relationship between RhoGDI2 expression and clinicopathological characteristics in PC.

\begin{tabular}{|c|c|c|c|c|c|}
\hline \multirow[b]{2}{*}{ Variables } & \multirow[b]{2}{*}{ Cases } & \multicolumn{2}{|c|}{ RhoGDI2 } & \multirow[b]{2}{*}{$\chi^{2}$} & \multirow[b]{2}{*}{ P-value } \\
\hline & & Negative & Positive & & \\
\hline Gender & & & & 0.506 & 0.625 \\
\hline Male & 48 & 16 & 32 & & \\
\hline Female & 29 & 12 & 17 & & \\
\hline Age (years) & & & & 0.430 & 0.632 \\
\hline$\leq 65$ & 45 & 15 & 30 & & \\
\hline$>65$ & 32 & 13 & 19 & & \\
\hline Tumor location & & & & 0.075 & 0.807 \\
\hline Head & 51 & 18 & 33 & & \\
\hline Body and tail & 26 & 10 & 16 & & \\
\hline Tumor size $(\mathrm{cm})$ & & & & 0.229 & 0.641 \\
\hline$\leq 2$ & 33 & 13 & 20 & & \\
\hline$>2$ & 44 & 15 & 29 & & \\
\hline Differentiation & & & & 0.003 & 0.999 \\
\hline Well & 22 & 8 & 14 & & \\
\hline Moderate & 25 & 9 & 16 & & \\
\hline Poor & 30 & 11 & 19 & & \\
\hline Clinical stage & & & & 19.983 & $0.008^{\mathrm{a}}$ \\
\hline I & 23 & 17 & 6 & & \\
\hline II & 54 & 11 & 43 & & \\
\hline $\begin{array}{l}\text { Lymph node } \\
\text { metastasis }\end{array}$ & & & & 16.418 & $0.013^{\mathrm{a}}$ \\
\hline Yes & 40 & 6 & 34 & & \\
\hline No & 37 & 22 & 15 & & \\
\hline
\end{tabular}

${ }^{\mathrm{a}} \mathrm{P}<0.01$.

was $100 \%$. After 3 years of follow up, only 8 of $77(10.4 \%)$ patients were alive and 69 patients $(89.6 \%)$ were deceased. The median survival time was 20 months (RhoGDI2-negative expression) and 11 months (RhoGDI2-positive expression),

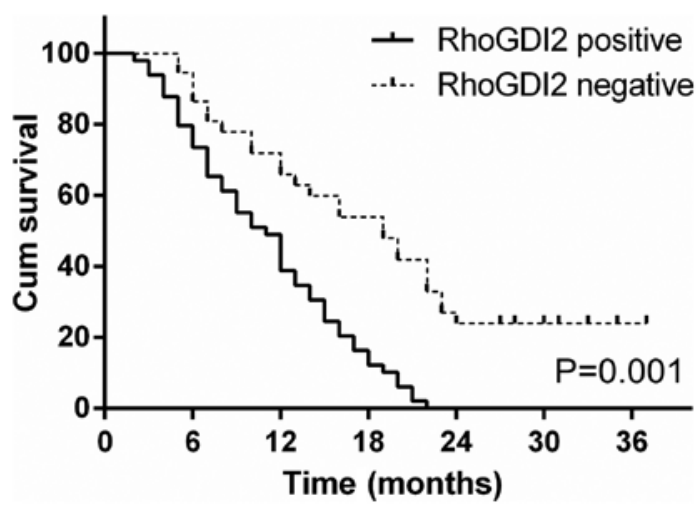

Figure 3. Survival curves of RhoGDI2 expression in PC patients. Survival of $77 \mathrm{PC}$ patients with different RhoGDI2 expression is shown. The survival rate for PC patients in the RhoGDI2-positive expression group was significantly lower than that for patients in the RhoGDI2-negative expression group (log-rank test, $\mathrm{P}=0.001$ ).

respectively. Kaplan-Meier curve assessment showed that the patients with RhoGDI2-negative expression had a significantly longer survival time than those with a RhoGDI2-positive expression (log-rank test, $\mathrm{P}=0.001$, Fig. 3).

The univariate analysis results revealed that RhoGDI2 expression $(\mathrm{P}=0.003)$, clinical stage $(\mathrm{P}=0.007)$ and lymph-node metastasis $(\mathrm{P}=0.006)$ were closely correlated with patient survival time (Table II). RhoGDI2 expression was closely associated with clinical stage and lymph-node metastasis. Thus, we used RhoGDI2 expression as the grouping variable, while clinical stage and lymph-node metastasis were considered the subgrouping variables. Stratified analysis showed that survival time of the RhoGDI2-positive expression group was significantly shorter than that of the RhoGDI2-negative expression group in the different subgroup levels $(\mathrm{P}<0.01$, Table III). The multivariate analysis results revealed that RhoGDI2 expression is one of the independent prognostic factors by Cox proportional hazards model ( $\mathrm{P}=0.008$, Table IV). The results indicated that the overexpression of RhoGDI2 was correlated with poor prognosis.

RhoGDI2 expression correlated with E-cadherin expression in PC tissues. E-cadherin is involved in epithelial to 
Table II. Univariate analysis of survival time of PC patients (Kaplan-Meier).

\begin{tabular}{|c|c|c|c|}
\hline Variables & Cases & $\begin{array}{l}\text { Average survival } \\
\text { period (months) }\end{array}$ & P-value \\
\hline Gender & & & 0.887 \\
\hline Male & 48 & $14.4 \pm 1.2$ & \\
\hline Female & 29 & $14.9 \pm 1.5$ & \\
\hline Age (years) & & & 0.495 \\
\hline$\leq 65$ & 45 & $14.2 \pm 1.1$ & \\
\hline$>65$ & 32 & $15.2 \pm 1.6$ & \\
\hline Tumor location & & & 0.379 \\
\hline Head & 51 & $15.1 \pm 1.2$ & \\
\hline Body and tail & 26 & $13.6 \pm 1.4$ & \\
\hline Tumor size (cm) & & & 0.131 \\
\hline$\leq 2$ & 33 & $13.0 \pm 1.3$ & \\
\hline$>2$ & 44 & $15.8 \pm 1.3$ & \\
\hline Differentiation & & & 0.178 \\
\hline Well & 22 & $12.5 \pm 1.5$ & \\
\hline Moderate & 25 & $14.2 \pm 1.5$ & \\
\hline Poor & 30 & $16.4 \pm 1.7$ & \\
\hline Clinical stage & & & $0.007^{\mathrm{a}}$ \\
\hline I & 23 & $20.7 \pm 1.9$ & \\
\hline II & 54 & $12.0 \pm 0.8$ & \\
\hline Lymph node & & & \\
\hline metastasis & & & $0.006^{\mathrm{a}}$ \\
\hline Yes & 40 & $11.1 \pm 1.0$ & \\
\hline No & 37 & $18.4 \pm 1.4$ & \\
\hline RhoGDI2 expression & & & $0.003^{\mathrm{a}}$ \\
\hline Positive & 49 & $11.1 \pm 0.8$ & \\
\hline Negative & 28 & $20.7 \pm 1.6$ & \\
\hline
\end{tabular}

mesenchymal transition (EMT), which is involved in invasion and metastasis in PC. To clarify the association between RhoGDI2 and E-cadherin, we firstly examined the expression of E-cadherin protein in 77 PC tissues by IHC (Fig 4). Data of the statistical analysis suggested that the expression of
Table IV. Multivariate analysis of prognostic markers in PC patients.

\begin{tabular}{lccc}
\hline Variables & HR & $95 \%$ CI & P-value \\
\hline Gender & 0.966 & $0.591-1.581$ & 0.892 \\
Age & 0.956 & $0.585-1.562$ & 0.858 \\
Tumor location & 0.677 & $0.404-1.136$ & 0.140 \\
Tumor size & 2.170 & $1.274-3.697$ & 0.004 \\
Differentiation & 1.174 & $0.680-2.028$ & 0.565 \\
Clinical stage & 0.491 & $0.254-0.948$ & $0.034^{\mathrm{a}}$ \\
Lymph-node metastasis & 1.889 & $1.085-3.288$ & $0.025^{\mathrm{a}}$ \\
RhoGDI2 expression & 3.344 & $1.718-6.505$ & $0.008^{\mathrm{a}}$ \\
\hline
\end{tabular}

${ }^{\mathrm{a}} \mathrm{P}<0.05$

Table V. Correlation between RhoGDI2 and E-cadherin expression in PC.

\begin{tabular}{lcccc}
\hline & \multicolumn{2}{c}{ RhoGDI2 expression } & & \\
\cline { 2 - 3 } $\begin{array}{l}\text { E-cadherin } \\
\text { expression }\end{array}$ & Positive (n) & Negative (n) & r & P-value \\
\hline Positive (n) & 6 & 21 & & \\
Negative (n) & 43 & 7 & -0.633 & $0.002^{\mathrm{a}}$ \\
\hline${ }^{\mathrm{a}}<0.01$. & & & & \\
\hline
\end{tabular}

RhoGDI2 was negatively correlated with the expression of E-cadherin in PC tissues $(\mathrm{P}=0.002$, Table $\mathrm{V})$.

\section{Discussion}

In this study, we examined the expression of RhoGDI2 in 30 matched clinical fresh tissues and 77 cases of paraffinembedded PC tissues. The results show that RhoGDI2 was overexpressed in PC tissues at mRNA and protein levels, and that RhoGDI2 expression was correlated with clinical stage, lymph-node metastasis and vascular invasion. Additionally, RhoGDI2 was one of the independent prognostic factors. We also found that the expression of RhoGDI2 was negatively correlated with the expression of E-cadherin in PC tissues.

Table III. Stratified analysis of related prognostic markers of PC patients.

\begin{tabular}{|c|c|c|c|c|c|}
\hline \multirow[b]{2}{*}{ Group } & \multirow[b]{2}{*}{ Cases } & \multicolumn{2}{|c|}{ Clinical stage } & \multicolumn{2}{|c|}{ Lymph-node metastasis } \\
\hline & & $\mathrm{I}$ & II & No & Yes \\
\hline RhoGDI2 positive & 49 & $16.8 \pm 5.3$ & $10.7 \pm 0.8$ & $15.8 \pm 2.3$ & $9.9 \pm 0.8$ \\
\hline RhoGDI2 negative & 28 & $22.1 \pm 1.8$ & $17.3 \pm 2.3$ & $20.2 \pm 1.7$ & $17.7 \pm 3.4$ \\
\hline $\mathrm{P}$-value & & 0.696 & $0.003^{\mathrm{a}}$ & 0.164 & $0.005^{\mathrm{a}}$ \\
\hline
\end{tabular}

${ }^{\mathrm{a}} \mathrm{P}<0.01$ 

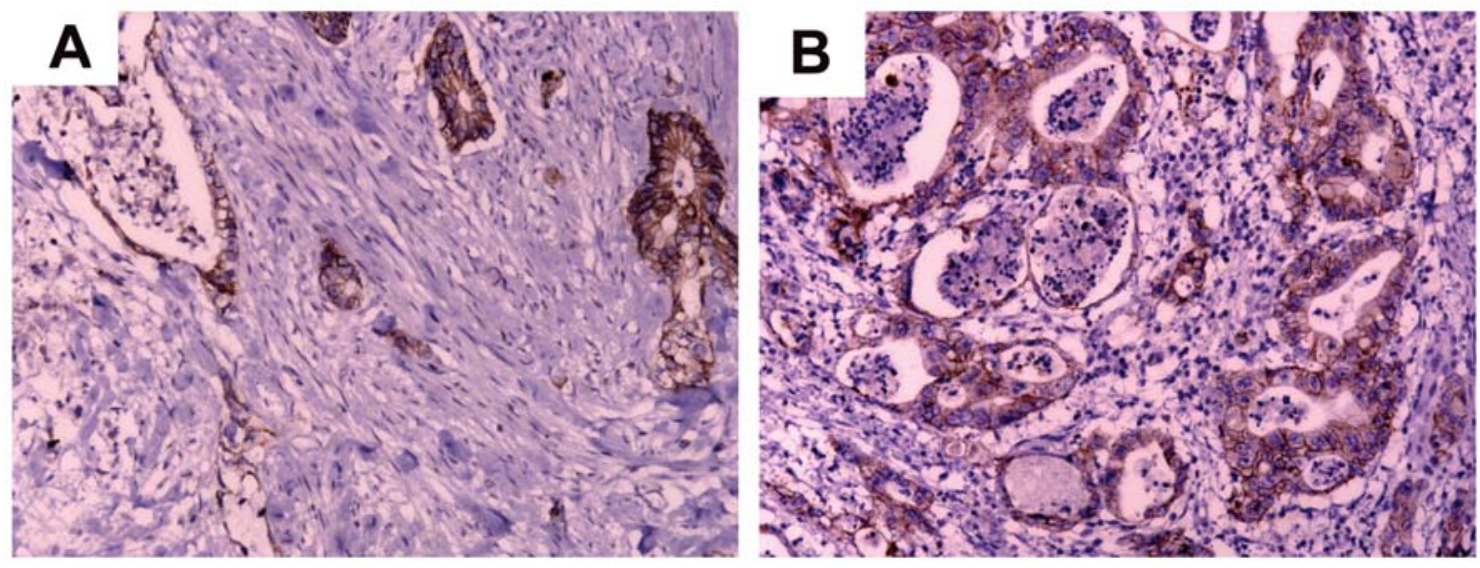

Figure 4. Representative immunohistochemical staining of E-cadherin in the two groups of PC tissues with (A) RhoGDI2-positive and (B) RhoGDI2-negative expression. Original magnification, x200.

These findings suggest that the upregulation of RhoGDI2 is involved in the progression and prognosis of PC.

RhoGDI2, also known as D4-GDI or LyGDI, has been identified as a regulator of Rho GTPases, which play important roles in cell motility, invasion and metastasis $(14,15)$. RhoGDI2 was preferentially expressed in hematopoietic tissues, predominantly in B and T lymphocytes (16). However, accumulating evidence reveals that RhoGDI2 is also aberrantly expressed in human cancers. In the majority of studies, RhoGDI2 has been shown to promote tumor cell invasion, angiogenesis and metastasis, such as in lung and gastric cancer $(17,18)$. However, it can function as a metastasis-suppressor gene in bladder cancer and Hodgkin's lymphoma $(19,20)$. Our results indicate that RhoGDI2 was overexpressed in PC and associated with clinicopathological characteristics of PC patients, including clinical stage and lymph-node metastasis. The conflicting role of RhoGDI2 may result from the dual roles of RhoGDI2 in the regulation of activities of Rho GTPases during cancer progression. RhoGDI2 binds the majority of Rho GTPases in the cytoplasm, maintaining Rho in an inactive form and inducing the disruption of Rho-dependent cell motility $(21,22)$. On the other hand, RhoGDI2 acted as an escort protein directing Rho GTPases to the membrane and is associated with active forms of Rho, Rac and Cdc42, maintaining them in an active form $(23,24)$. However, the exact mechanisms remain to be determined.

In this study, we have demonstrated that RhoGDI2 expression is one of the independent prognostic factors in PC, and overexpression of RhoGDI2 was correlated with poor prognosis. Stratified analysis of survival time showed that in lymph-node positive patients, the prognosis of PC with RhoGDI2-positive expression was worse than that of ones with RhoGDI2-negative expression. Similar results were obtained in stage II of PC patients with different RhoGDI2 expression. This finding indicated that, for PC patients with lymph-node metastasis and clinical stage II, we may draw up individualized gene therapy and evaluate prognosis by detecting RhoGDI2 expression.

EMT is an essential cell mechanism during tumor progression, which induces tumor cell migration, invasion and metastasis (25). In all EMT processes, cells lose the expression of a cell-to-cell adhesion molecule known as E-cadherin, which functions as a molecular glue that attaches cells to one another (26). To clarify the underlying mechanism of RhoGDI 2 in the progression of tumor invasion and metastasis, we also investigated the relationship between and in PC tissues. Our results (data not shown) indicated E-cadherin was downregulated in PC tissues and was negatively correlated with the expression of RhoGDI2. In our previous study, RhoGDI2 was known to promote PC cell invasion and migration in vitro, but to the best of our knowledge, this is the first study showing that RhoGDI2 expression was correlated with E-cadherin expression in PC tissues.

In conclusion, our study has demonstrated that the overexpression of RhoGDI2 was associated with PC progression and played an important role in predicting the prognosis of PC patients. Moreover, upregulation of RhoGDI2 was associated with reversal of E-cadherin expression in PC tissues. These findings indicate that targeting RhoGDI2 may be a useful strategy for inhibiting the invasion and metastasis of PC.

\section{Acknowledgements}

This study was supported by the Project of Nature Science Foundation of China (81201905), Nature Science Research Grants at the University of Jiangsu Province of P.R. China (14KJB320019) as well as the Project of Medical Research of Jiangsu Province (Q201402).

\section{References}

1. Siegel R, Naishadham D and Jemal A: Cancer statistics, 2013. CA Cancer J Clin 63: 11-30, 2013.

2. Vincent A, Herman J, Schulick R, Hruban RH and Goggins M: Pancreatic cancer. Lancet 378: 607-620, 2011.

3. Vega FM and Ridley AJ: Rho GTPases in cancer cell biology. FEBS Lett 582: 2093-2101, 2008.

4. Reymond N, Riou P and Ridley AJ: Rho GTPases and cancer cell transendothelial migration. Methods Mol Biol 827: 123-142, 2012.

5. Garcia-Mata R, Boulter E and Burridge K: The 'invisible hand': regulation of RHO GTPases by RHOGDIs. Nat Rev Mol Cell Biol 12: 493-504, 2011.

6. Cho HJ, Baek KE and Yoo J: RhoGDI2 as a therapeutic target in cancer. Expert Opin Ther Targets 14: 67-75, 2010.

7. Agarwal NK, Chen CH, Cho H, Boulbes DR, Spooner E and Sarbassov DD: Rictor regulates cell migration by suppressing RhoGDI2. Oncogene 32: 2521-2526, 2013. 
8. Griner EM and Theodorescu D: The faces and friends of RhoGDI2. Cancer Metastasis Rev 31: 519-528, 2012.

9. Yi B, Hu Y, Qin G, et al: Depletion of RhoGDI2 expression inhibits the ability of invasion and migration in pancreatic carcinoma. Int J Mol Med 34: 205-212, 2014

10. Rhim AD, Mirek ET, Aiello NM, et al: EMT and dissemination precede pancreatic tumor formation. Cell 148: 349-361, 2012

11. Kevans D, Wang LM, Sheahan K, et al: Epithelial-mesenchymal transition (EMT) protein expression in a cohort of stage II colorectal cancer patients with characterized tumor budding and mismatch repair protein status. Int J Surg Pathol 19: 751-760, 2011.

12. Matsuoka J, Yashiro M, Doi Y, et al: Hypoxia stimulates the EMT of gastric cancer cells through autocrine TGF $\beta$ signaling. PLoS One 8: e62310, 2013.

13. Burgess DJ: Breast cancer: Circulating and dynamic EMT. Nat Rev Cancer 13: 148, 2013.

14. Nitz MD, Harding MA and Theodorescu D: Invasion and metastasis models for studying RhoGDI2 in bladder cancer. Methods Enzymol 439: 219-233, 2008.

15. Li X, Wang J, Zhang X, Zeng Y, Liang L and Ding Y: Overexpression of RhoGDI2 correlates with tumor progression and poor prognosis in colorectal carcinoma. Ann Surg Oncol 19: $145-153,2012$

16. Scherle P, Behrens T and Staudt LM: Ly-GDI, a GDP-dissociation inhibitor of the RhoA GTP-binding protein, is expressed preferentially in lymphocytes. Proc Natl Acad Sci USA 90: 7568-7572, 1993.

17. Niu H, Li H, Xu C and He P: Expression profile of RhoGDI2 in lung cancers and role of RhoGDI2 in lung cancer metastasis. Oncol Rep 24: 465-471, 2010.
18. Cho HJ, Baek KE, Kim IK, et al: Proteomics-based strategy to delineate the molecular mechanisms of RhoGDI2-induced metastasis and drug resistance in gastric cancer. J Proteome Res 11: 2355-2364, 2012.

19. Theodorescu D, Sapinoso LM, Conaway MR, Oxford G, Hampton GM and Frierson HF Jr: Reduced expression of metastasis suppressor RhoGDI2 is associated with decreased survival for patients with bladder cancer. Clin Cancer Res 10: 3800-3806, 2004.

20. Ma L, Xu G, Sotnikova A, et al: Loss of expression of LyGDI (ARHGDIB), a rho GDP-dissociation inhibitor, in Hodgkin lymphoma. Br J Haematol 139: 217-223, 2007.

21. Dovas A and Couchman JR: RhoGDI: multiple functions in the regulation of Rho family GTPase activities. Biochem J 390: 1-9, 2005.

22. DerMardirossian C and Bokoch GM: GDIs: central regulatory molecules in Rho GTPase activation. Trends Cell Biol 15: 356-363, 2005

23. Hart MJ, Maru Y,Leonard D, Witte ON, Evans T and Cerione RA: A GDP dissociation inhibitor that serves as a GTPase inhibitor for the Ras-like protein CDC42Hs. Science 258: 812-815, 1992.

24. Chuang TH, Xu X, Knaus UG, Hart MJ and Bokoch GM: GDP dissociation inhibitor prevents intrinsic and GTPase activating protein-stimulated GTP hydrolysis by the Rac GTP-binding protein. J Biol Chem 268: 775-778, 1993.

25. Yilmaz M and Christofori G: EMT, the cytoskeleton, and cancer cell invasion. Cancer Metastasis Rev 28: 15-33, 2009.

26. Theys J, Jutten B, Habets R, et al: E-Cadherin loss associated with EMT promotes radioresistance in human tumor cells. Radiother Oncol 99: 392-397, 2011. 